\title{
Functional Impact of An ADHD-Associated DIRAS2 Promoter Polymorphism
}

\author{
Lena Grünewald*, , Elisabeth Toverud Landaas², Julia Geissler ${ }^{3}$, Heike Weber', Carina Quast ${ }^{4}$, Simone Röh ${ }^{4}$, \\ Christoph Schartner ${ }^{5}$, Klaus-Peter Lesch ${ }^{5}$, Marcel Romanos ${ }^{3}$, Sarah Kittel-Schneider', Elisabeth Binder ${ }^{4,6}$ and \\ Andreas Reif' \\ 'Department of Psychiatry, Psychosomatics and Psychotherapy, Translational Psychiatry, University Hospital Frankfurt, Frankfurt, Germany; \\ ${ }^{2}$ Department of Biomedicine, K.G. Jebsen Centre for Neuropsychiatric Disorders, University of Bergen, Bergen, Norway; ${ }^{3}$ Department of Child and \\ Adolescent Psychiatry, Psychosomatics and Psychotherapy, University of Würzburg, Würzburg, Germany; ${ }^{4}$ Department of Translational Research in \\ Psychiatry, Max Planck Institute of Psychiatry, Munich, Germany; ${ }^{5}$ Department of Psychiatry, Psychosomatics and Psychotherapy, Molecular \\ Psychiatry Laboratory of Translational Neuroscience, University of Würzburg, Würzburg, Germany; ${ }^{6}$ Department of Psychiatry and Behavioral \\ Sciences, Emory University School of Medicine, Atlanta, GA, USA
}

\begin{abstract}
The DIRAS2 gene is coding for a small Ras GTPase with so far unknown function. In a previous study, we described the association of DIRAS2 rs I I 2005, as well as a haplotype containing this polymorphism and located in the promoter region of this gene with attentiondeficit/hyperactivity disorder (ADHD). In the present study, we searched for rare variants within or near the DIRAS2 gene that might be associated with ADHD using next-generation sequencing. As we were not able to detect any rare variants associated with the disease, we sought to establish a functional role of DIRAS2 rsI4I2005 on the molecular or systems level. First, we investigated whether it has an influence on gene expression by means of a luciferase-based promoter assay. We could demonstrate that the minor risk allele goes along with the increased expression of the reporter gene. Next, we aimed to identify differences in response inhibition between risk-allele and non-risk allele carriers in children suffering from ADHD and healthy control individuals by analyzing event-related potentials in the electroencephalogram during a Go/NoGo task. Risk-allele carriers showed a changed NoGo anteriorization. Therefore, our results suggest an impact of the investigated polymorphism on the prefrontal response control in children with ADHD. These results imply that the promoter polymorphism is indeed the associated as well as in itself a causal variant. Further research is thus warranted to clarify the mechanisms linking DIRAS2 to ADHD.

Neuropsychopharmacology (2016) 4I, 3025-303I; doi: I 0.1038/npp.2016.1 I3; published online 27 July 2016
\end{abstract}

\section{INTRODUCTION}

Attention-deficit/hyperactivity disorder (ADHD) is neurodevelopmental in nature and characterized by inattention, hyperactivity, and increased impulsivity. The disorder is common among children, with an estimated prevalence of up to 5\% (Polanczyk et al, 2007), and over the last decades, it has become evident that a majority of the patients experience significant symptoms also in adulthood (Faraone et al, 2005). The disorder is frequently observed to aggregate in families, and the heritability of childhood ADHD (cADHD) has been estimated to around $76 \%$, giving evidence of a strong genetic component (Faraone et al, 2005). Many genetic loci have been reported to be associated with ADHD, but only few of these findings have been successfully replicated. Accordingly, overall findings are inconsistent, and most of the genetic

\footnotetext{
*Correspondence: Dr L Grünewald, Department of Psychiatry, Translational Psychiatry, Psychosomatics and Psychotherapy, Translational Psychiatry, University Hospital Frankfurt, Heinrich-Hoffmann-Str 10, Frankfurt D-60528, Germany, Tel: +49 696301 83664, Fax: +49 69 630। 81674, E-mail: lena.weissflog@kgu.de

Received 21 April 2016; revised 20 June 20 16; accepted 24 June 2016; accepted article preview online I July 2016
}

contribution remains to be explained (Franke et al, 2009; Gizer et al, 2009).

One of the genes that have shown promising results as ADHD risk candidates is the GTP-binding Ras-like protein 2 gene (DIRAS2). Attention was first drawn to this gene when its locus on chromosome 9q22.2 was implicated in two cADHD-linkage scans from Germany (Romanos et al, 2008) and the UK (Asherson et al, 2008). Further corroborating evidence came from our previous genome-wide association study (GWAS) on adult ADHD (aADHD) patients, recruited from the same catchment area as the German linkage study (Lesch et al, 2008). Two of the 500 top ranking single nucleotide polymorphisms (SNPs) were located in an intergenic region on chromosome 9q22.2. The neighboring gene found most likely to be involved in the aetiopathogenesis of ADHD was DIRAS2, because, in contrast to the other neighbor $(S Y K)$, it is expressed in the brain (Kontani et al, 2002). On the basis of these reports, we further examined a potential association between tagging SNPs in DIRAS2 and aADHD in a German sample of 600 patients and 420 controls, as well as European replication sample of 1035 patients and 1381 controls (Reif et al, 2011). Four SNPs and two haplotype blocks showed significant results $(p<0.05)$ in 
the German sample. Meta-analyses with the replication sample showed consistent and significant effects for the promoter SNP rs1412005 (odds ratio $(\mathrm{OR})=1.12 ; p=0.04$ ), and for a haplotype comprising six SNPs including the risk allele of $\mathrm{rs} 1412005$ (OR $=1.45 ; p=0.0003)$.

In the present study, we follow up these previous findings with the objective of coming closer to an understanding of the mechanisms behind the observed associations between ADHD and DIRAS2. Our first aim was to investigate whether the promoter SNP rs1412005 is indeed the causal variant, or if the reported association is rather a 'synthetic association' observed due to rare causal variants located in same haploblock as the common variant (Dickson et al, 2010). To search for unknown and rare (minor-allele frequency (MAF) <0.05) variants, we used next-generation sequencing (NGS) technology on DNA from aADHD patients and controls. To test for a regulatory function of the SNP on gene expression, we performed a luciferase reporter assay. To study the functional impact of the polymorphism on the systems level, especially during neurodevelopment (and assuming a stronger functional effect in children), we explored whether the DIRAS2 rs1412005 risk variant is also associated with the altered response inhibition, a putative endophenotype of ADHD. To do so, we measured inhibitory performance and alterations in brain activation patterns by analyzing event-related potentials (ERPs) in the electroencephalogram (EEG) of 62 children suffering from ADHD, as well as 36 matched controls during a Go/NoGo task (Rosvold et al, 1956).

\section{MATERIALS AND METHODS}

\section{Next-Generation Sequencing}

Samples. The patient sample consisted of 300 aADHD patients (99 males and 201 females; 176 combined, 97 inattentive, and 21 hyperactive-impulsive subtype) ascertained at the Department of Psychiatry, University of Würzburg. The age at inclusion ranged from 18 to 70 years $($ mean $=33$ years; $S D=9.8)$. The inclusion criteria was aADHD according to the DSM-IV criteria; onset before 7 years of age via retrospective diagnosis, life-long persistence and current diagnosis, and additionally an intelligence quotient (IQ) level $>80$ and absence of bipolar affective disorder. DNA from 300 healthy individuals (98 males and 202 females) recruited from the Lower Franconia Area of Germany served as the control sample. Controls were matched with the patients for sex and the mean age at recruitment was 24 years $(\mathrm{SD}=4.2)$. All included individuals were Caucasians. Written informed consent was obtained from all participants and the study was approved by the Ethics Committee of the University of Würzburg. The overall sample has previously been described in greater detail (Franke et al, 2009; Jacob et al, 2007).

DNA amplification and sequencing. Equimolar amounts of DNA from either 50 patients or 50 controls were pooled prior to the amplification. The exons of the DIRAS2 gene including exon-intron boundaries and untranslated regions including the promoter region, were amplified by polymerase chain reactions (PCRs) using either the iProof High Fidelity DNA Polymerase (Bio-Rad Laboratories, München, Germany) or the SequalPrep Long PCR Kit from Invitrogen (Invitrogen; see Supplementary Table 1 for primer sequences). Equimolar amounts of the amplicons from two patient or control pools were combined. Samples were prepared for the sequencing run performed on the Life Technologies SOLiD 3+ sequencer following the manufacturer's instructions. Bead production and enrichment was carried out manually as a full-scale reaction. For the emulsion PCRs, a final concentration of $0.5 \mathrm{pm}$ of each library was used as DNA template. Bead enrichment resulted in 87 to 110 million beads per library. The SOLiD sequencing run was performed using SOLiD Opti sequencing chemistry for a single F3 Tag with a read length of $50 \mathrm{bp}$.

Data analysis: variant detection and annotation. The raw reads generated by the SOLiD run underwent a two-step quality control (QC) procedure. First, reads were trimmed dynamically, eliminating all bases succeeding and including the fifth base of insufficient quality (phred-like quality value $\leqslant 10)$. Second, trimmed reads consisting of $<30$ bases in length were excluded. The reads overcoming the QC were aligned using BWA version 0.6.2 ( $\mathrm{Li}$ and Durbin, 2009) to chromosome 9 of the human genome (hg19), allowing a maximum of four mismatches. For additional confirmation, we used SHRIMP version 2.2.2 (David et al, 2011) as the alternative alignment strategy. To perform single nucleotide variation (SNV) calling, we used vipR (http://sourceforge. net/projects/htsvipr/) (Altmann et al, 2011). vipR compares the number of base calls for the minor allele at a given position obtained from a given pool with the minor allele calls at the same position obtained from the remaining pools. Using the Skellam distribution, vipR assesses the likelihood of the base calls originating from sequencing errors. Only if the likelihood is sufficiently small, the minor allele at that position is reported as a SNV. Only positions with coverage of at least 5000 at a given base position in each pool were included for variant calling, resulting in an exclusion rate of $10 \%$ of the sequenced region. SNV annotation was performed using ANNOVAR (Annotation Variant; http:// www.openbioinformatics.org/annovar/) (Wang et al, 2010).

Genotyping and replication of sequencing results. To validate the results of the SOLiD sequencing, non-pooled DNA was genotyped using TaqMan Assays (Life Technologies) following the manufacturer's instructions and, in one case where this was not feasible (rs10993608), using Sanger sequencing (Eurofins, Ebersberg, Germany). Primers and sequences are available on request. Genotyping of the DIRAS2 SNP rs1412005 was performed with a Taq-Man assay (assay ID C__3069317_10) according to the manufacturer's specifications (Applied Biosystems) with a call rate of $93.3 \%$. We analyzed the data using a dominant model, comparing risk-allele carriers (genotypes TT and GT) and participants without the risk allele (genotype GG).

\section{Luciferase Reporter Assay}

Complementary oligonucleotides containing the risk or the non-risk allele of the promoter SNP, and a HindIII or a NheI restriction enzyme overhang (Supplementary Table 1 for the sequences) have been annealed and cloned into the pGL4.23 minimal promoter firefly luciferase 2 gene vector 
(Promega, Mannheim, Germany). The human neuroblastoma cells SH-SY5Y (European Collection of Cell Cultures, Salisbury, UK) were seeded at a density of $5 \times 10^{4}$ cells/well into 24-well culture plates. Twenty-four hours after seeding, cells were transfected with $75 \mathrm{ng}$ of the pGL4.74 vector (Promega, Mannheim, Germany) coding for the renilla luciferase under the control of a HSV-TK promoter and with $675 \mathrm{ng}$ of the pGL4.23 constructs following the TransFast Transfection Kit protocol (Promega, Mannheim, Germany). Forty-eight hours post transfection, the luciferase activities were measured on the GloMax Multi+ Detection System luminometer (Promega, Mannheim, Germany). Measurements were carried out in triplicates. Each experimental assay was performed three times.

Background signals were subtracted from the raw data, and the quotients between firefly and renilla luciferase activity were calculated for normalization. $Z$-scores were calculated for the minor and major alleles, adjusting for daily variations between the measurements. Finally, a two-sided Welch's two-sample $t$-test was performed using the software $\mathrm{R}$ version 2.15.1 (www.r-project.org).

Transcription factor-binding sites that would be altered by the presence of the different nucleotides at rs1412005 were searched using the FuncPred tool (http://manticore.niehs. nih.gov). With the aim of examining if the reported transcription factors are actually expressed in SH-SY5Y cells, an expression study was performed. RNA was isolated from SH-SY5Y cells using the RNeasy Plus Mini Kit (Qiagen, Hilden, Germany) and reversely transcribed into complementary DNA (cDNA) using the iScript cDNA synthesis kit (Bio-Rad Laboratories). Quantitative real-time PCR was run on a CFX384 Real-Time PCR Detection System using iQ SYBR Green SuperMix (Bio-Rad Laboratories) and QuantiTect Primer Assays (Qiagen, Hilden, Germany).

\section{Functional Correlates in the EEG}

Sample. The investigated sample comprised 62 cADHD patients of which 55 had the combined subtype and 7 the inattentive subtype of the disease. Of these 62 patients, 30 were unmedicated (mean age $=10.06 \pm 0.48$ years; age range 5-17 years; 12 females and 20 males; 4 inattentive subtype; 26 combined subtype), whereas 32 received psychostimulants (mean age $=10.52 \pm 0.42$ years; age range $7-16$ years; 6 females and 25 males; 3 inattentive subtype; 29 combined subtype). The patients were recruited from the Clinic for Child and Adolescent Psychiatry at the university hospital in Würzburg. The control group comprised 36 matched

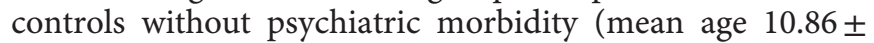
0.56 years; age range $6-17 ; 18$ females and 18 males). Exclusion criteria were IQ $<70$ for all the participants, and for controls, the presence of psychiatric disorders, whereas for ADHD patients, patients with conditions that interfered with EEG recordings, like Tourette's syndrome, severe motor tics, or epilepsy, where excluded.

Cued continuous performance task. Participants were seated in front of a computer screen in a quiet darkened room and were instructed to press a button as quickly as possible whenever the letter $\mathrm{O}$ was followed by the letter $\mathrm{X}$ (Go trials), but to not react to any other combination of the stimuli. Stimuli were presented for $200 \mathrm{~ms}$, with an interstimulus interval of $1650 \mathrm{~ms}$.

EEG recording. We recorded a 21-channel EEG placed according to the international 10-20 system (Fp1, Fpz, Fp2, F3, Fz, F4, F7, F8, T3, T4, C3, Cz, C4, T5, T6, P3, Pz, P4, O1, Oz, O2; (Jasper, 1958)) with a 32-channel DC amplifier (Brain-Star System, Erlangen, Germany) and the software Vision Recorder (Brain Products $\mathrm{GmbH}$, Munich, Germany) at a sampling rate of $1000 \mathrm{~Hz}$, a bandpass between 0.1 and $100 \mathrm{~Hz}$, and a $50 \mathrm{~Hz}$ notch filter. The recording reference was placed between $\mathrm{Fz}$ and $\mathrm{Cz}$.

Data analysis. For offline data analysis, the Vision Recorder software (Brain Products $\mathrm{GmbH}$, Munich, Germany) was used. A band-pass filter $(0.1-50 \mathrm{~Hz})$ was applied and the data were re-referenced to an average reference. Blink detection was accomplished with the algorithm by Gratton and Coles (Gratton and Coles, 1989). After segmentation into Go (correct response) and NoGo (successful inhibition) trials, segments containing signals exceeding $\pm 100 \mu \mathrm{V}$ were rejected, leaving a minimum of 15 valid epochs. P300 peaks were semiautomatically determined at $\mathrm{Pz}$ for $\mathrm{Go}$ and at $\mathrm{Cz}$ for NoGo in the Global Field Power ERP. The location of the centre of the brain-electric field (centroid) was calculated with the weighted centroids method (Lehmann and Skrandies, 1980) for that precise point in time. NoGo anteriorization (NGA) was computed by subtracting the NoGo from the Go centroid location, with higher values indicating better anteriorization.

For statistical analyses, we used SPSS version 18. As preliminary results indicated strong effects of DIRAS2 genotypes in an ADHD sample, we conducted separate analyses for functional DIRAS2 effects on ADHD children and adolescents (mean age $10.38 \pm 3.0$ years), and matched healthy controls (mean age $10.81 \pm 0.54$ years) without psychiatric morbidity. As behavioral parameters were not normally distributed, they were analyzed nonparametrically with Kruskal-Wallis or Wilcoxon's tests, respectively, whereas all electrophysiological data were examined in analyses of variance with a significance level of $p=0.05$.

\section{RESULTS}

\section{Sequencing}

Pooled NGS revealed eight variants within the DIRAS2 coding sequences or flanking regions to differ significantly between ADHD patients and healthy controls $(0.047<p<$ $\left.4.2 \times 10^{-5}\right)$. The positions of the variants on chromosome 9 , minor and major alleles, MAFs in patients and controls, and $p$-values are given in Table 1. rs10993608 was especially intriguing, as the minor allele was exclusively present in ADHD patients according to the NGS data.

To verify the sequencing results, the DNA of ADHD patients and controls that had been sequenced, was individually genotyped using TaqMan probes specific for six of the eight identified variants (rs10993608, rs80274505, rs6479611, and variants at positions 93402517, 93409910, and 93410462; the remaining two were omitted owing to their borderline significance not surviving correction for multiple testing). The chromosomal positions of the investigated variants and 
Table I Positions of the Variants that Differ Significantly Between ADHD Patients and Healthy Controls Within or Near the DIRAS2 Gene on Chromosome 9, Minor and Major Alleles, Minor Allele Frequencies (MAF) in Patients and Controls, and p-values

\begin{tabular}{|c|c|c|c|c|c|c|c|}
\hline Chromosomal position & Region & SNP ID & REF & ALT & MAF cases & MAF controls & $P$-value \\
\hline 93369678 & Downstream & rs10993608 & G & A & 0.076 & 0.001 & 0.000042 \\
\hline 93371890 & Downstream & rs80274505 & $\mathrm{T}$ & G & 0.002 & 0.036 & 0.023378 \\
\hline 93402517 & Intronic & - & $\mathrm{T}$ & A & 0.756 & 0.617 & 0.003869 \\
\hline 93403987 & Intronic & - & G & A & 0.374 & 0.267 & 0.047137 \\
\hline 93404249 & Intronic & rs647961I & A & G & 0.431 & 0.610 & 0.000253 \\
\hline 93410463 & Upstream & - & C & $\mathrm{T}$ & 0.728 & 0.618 & 0.041002 \\
\hline
\end{tabular}

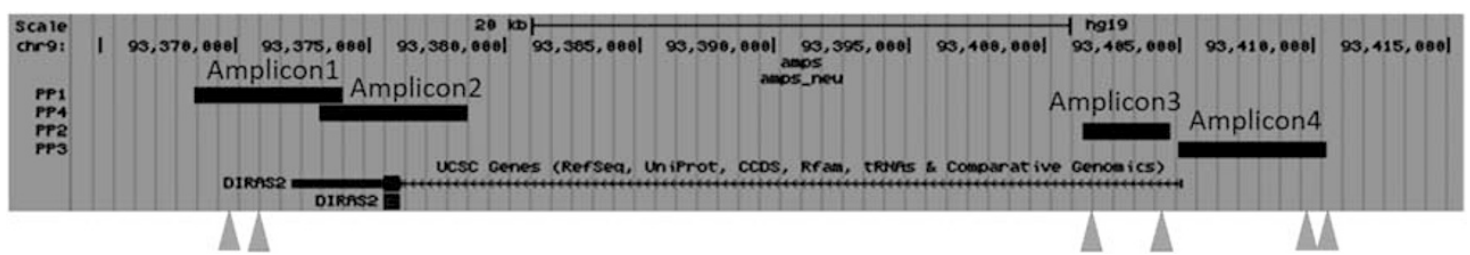

Figure I Schematic of the DIRAS2 gene taken from the UCSC Genome Browser (http://genome.ucsc.edu/) showing the positions of the amplicons used for NGS (black bars) and the variants investigated in the replication (red triangles). A full color version of this figure is available at the Neuropsychopharmacology journal online.

an alignment of the amplicons generated for the NGS are shown in Figure 1. The TaqMan assays for rs10993608 and the variant at position 93410462 failed to produce any reliable results. The four assays genotyping rs80274505, rs6479611 and the variants at positions 93402517 and 93409910 however generated reliable data. In contrast to NGS results, genotypes were not significantly different between the cases and the controls. As the genotyping assay for rs10993608 failed, DNA fragments containing that variant were additionally investigated by Sanger sequencing. The minor allele suggested by NGS could not be detected. Also, the total count of minor alleles was not significantly higher for ADHD cases than for controls when performing individual genotyping (data not shown). As we could not detect rare variants associated with ADHD, we continued to study the functional effects of rs1412005 on the molecular and systems level.

\section{Luciferase Assay}

The firefly luciferase activity measurements were in the range $0.4 \times 10^{5}-2.7 \times 10^{5}$ relative luminescence units after subtraction of the background signals. The $t$-test for the $z$-score of the luminescence by rs1412005 risk allele gave a $t$-value of -5.648 , and the corresponding $p$-value for the difference between the constructs containing the major and minor allele was $4.2 \times 10^{-5}$ (Figure 2).

Using the National Institute of Environmental Health Sciences homepage's SNP function prediction tool, rs1412005 was found to alter the binding sites of the androgen receptor, the hepatocyte nuclear factor 1 homeobox A (HNF1A), the cyclooxygenase-1 (COX1), the paired box protein Pax-6, cutlike homeobox 1 (CUX1), and the peroxisome proliferator-

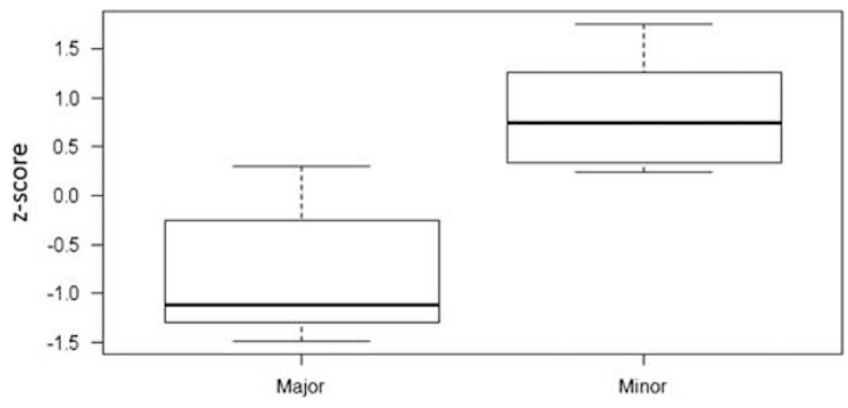

Figure 2 Boxplot showing the combined $z$-scores for the three luciferase activity measurements in SH-SY5Y cells of the DIRAS2 SNP rs I 4 I2005. The luciferase activity is significantly higher $\left(p=4.207 \times 10^{-5}\right)$ in cells transfected with the constructs containing the minor allele of the DIRAS2 promoter polymorphism.

activated receptor gamma (PPAR $\gamma)$. When analyzing cDNA from SH-SY5Y cells, we found that PPAR $\gamma$ and CUX1 were both expressed in these cells, whereas no expression of the other transcription factors was found.

\section{Response Inhibition: Behavioral and ERP Data}

Behavior. DIRAS2 genotype alone did not affect errors or reaction times in total sample or in individual diagnostic groups (all $p$-values $>0.240$ ).

Centroids. The repeated measures ANOVA comparing locations of Go and NoGo centroids for control subjects only yielded a main effect for condition, signifying more anterior centroids for NoGo trials $\left(F_{1,34}=10.240, p=0.003\right)$. In the $\mathrm{ADHD}$ group, this main effect $\left(F_{1},{ }_{58}=9.341\right.$, 


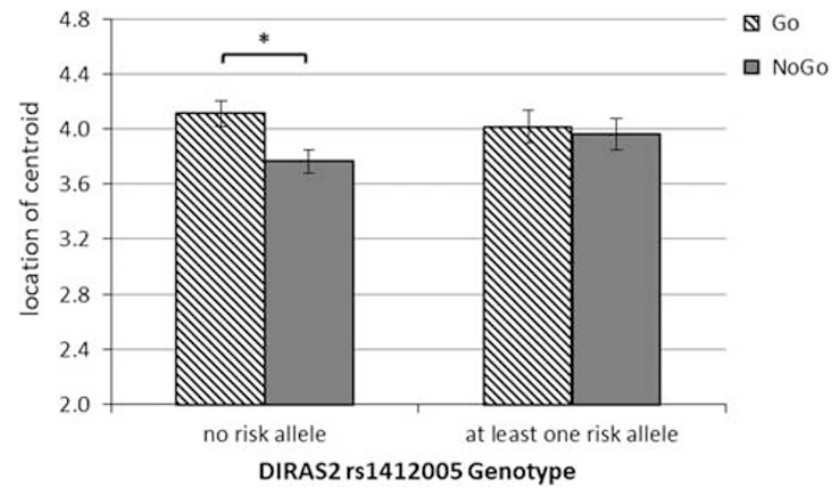

Figure 3 Position of the centroids in the Go/NoGo task in risk-allele carriers and non-risk allele carriers. In individuals lacking the risk allele of the DIRAS2 promoter polymorphism, no NoGo anteriorization could be found. Statistically significant difference is indicated by an asterisk $(p=0.00 \mathrm{I})$.

$p=0.003)$ was a result of an interaction of DIRAS2 genotype and condition $\left(F_{1}, 58=5.917, p=0.018\right)$. The difference between conditions in terms of centroid location was only observed in rs1412005 non-risk allele carriers $(p=0.001$; $n=32)$, but not in the risk-allele group $(p=0.539 ; n=30$; Figure 3). Testing for a group effect comparing medicated with unmedicated patients revealed no differences between groups $(p=0.484)$.

NoGo anteriorization. We found a main effect for DIRAS2 genotype in ADHD children $\left(F_{1,58}=5.917, p=0.018\right)$, but not in the control group $(p=0.869)$. ADHD individuals with at least one risk allele had a dampened NGA compared with those without the risk allele. The medication status did not influence NGA in patients $(p=0.986)$.

\section{DISCUSSION}

We here followed up on previous reports of DIRAS2 as an ADHD candidate gene, with the aim of better understanding the underlying mechanisms of the observed associations, particularly regarding the promoter SNP rs1412005. Our results show that the rs1412005 risk allele results in significantly higher gene expression as measured by luciferase activity. We found no rare variants in neither the coding nor the flanking regions of DIRAS2 likely to better explain the previously observed association signal. Further, we observed that the risk allele is associated with a diminished NGA in ADHD, despite normal behavioral performance.

Notwithstanding the results of our previous association studies, the possibility remains that there are rare variants in linkage disequilibrium with rs1412005, which are truly causal and thus represent what has been called 'synthetic associations' (Dickson et al, 2010). However, the idea that those synthetic associations caused by rare variants could explain the majority of GWAS results has been questioned (Wray et al, 2011) by reanalyzing real life GWAS data and by running additional simulations. Nevertheless, we first performed a NGS experiment yielding eight promising variants that were associated with disease. None of the association signals however survived replication attempts suggesting that these were false-positive results, emphasizing the need for verification of NGS data. As PCR amplification was used to generate specific DNA fragments for the sequencing, false positives could be introduced due to misincorporation of bases during this process. Such problems could be overcome by designing overlapping amplicons and accepting only consensus-variant calls (Meldrum et al, 2011). However, this strategy would lead to a considerable cost increase. As the patient and the control sample consisted of only 300 individuals each, we can evidently not rule out that there could be even rarer variants or variants in other populations. Nevertheless, our SNP association finding on rs1412005 would still be reliable. Thus, with a high level of confidence, we conclude that indeed rs1412005 is the causal variant associated with aADHD in our previous studies.

To establish a functional role for rs1412005, we performed a luciferase assay and demonstrated increased reporter gene expression when the minor allele was present, suggesting that the risk allele may lead to higher DIRAS2 expression. However, a luciferase promoter assay performed in a human cancer cell line only provides limited information of the processes in the human organism. Also, the association of DIRAS2 with aADHD is not only based on the promoter polymorphism as such, but also on the haplotype block containing it (Reif et al, 2011). As this haploblock spans $>5 \mathrm{~kb}$, it was not possible to clone the entire region into the luciferase reporter vector to test for expression changes owing to the full risk haplotype. As DIRAS2 is not expressed in human blood cells, attempts to determine DIRAS2 expression levels in human samples with the relevant genotypes did thus far not lead to reliable results.

According to the SNP function prediction tool used for detecting changes in transcription factor-binding sites, the promoter polymorphism may lead to altered binding sites of six transcription factors. However, only two of these, $P P A R \gamma$ and CUX1, were found to be expressed in the brain and also in the SH-SY5Y cells used for the luciferase assay, and therefore may be involved in the observed expression changes. To investigate whether PPAR $\gamma$ or CUX1 are crucial for the regulation of DIRAS2 expression, a knockdown of the transcription factors followed by luciferase assays will be performed in future experiments, thereby clarifying the molecular cascade leading to differential DIRAS2 expression.

The impact of the possible changes in DIRAS2 gene expression is difficult to predict, as the functions of the gene, or the Di-ras2 protein respectively, and the pathways via which they operate, are generally unknown. Di-ras2 is member of the Ras superfamily of small guanosine triphosphatases. Other members of the Ras superfamily are involved in the regulation of cell functions such as proliferation and differentiation (Wennerberg et al, 2005). If Di-ras2 possesses similar functions, this could be important in relation to ADHD being a neurodevelopmental disorder.

DIRAS2 has been found to be almost uniquely expressed in the brain. The expression is reported to be strongest in the cerebral cortex, the occipital pole, the frontal, and temporal lobes and the cerebellum (Kontani et al, 2002). Among these regions, for instance, the prefrontal cortex and the cerebellum are known to constitute core parts of the network regulating impulsive decision making and behavior, which are traits affected in ADHD. In line with this, several studies have suggested that Di-Ras2 is involved in cognitive processes and behavior. Seshadri et al (Seshadri et al, 2007) found DIRAS2 to be associated with the performance on the 
Boston naming test in a GWAS of the ageing brain and Wylie et al (Wylie et al, 2010) found a 13-fold increase in Diras2 expression in serotonergic neurons from the caudal hindbrain of mouse embryos, which could be indicative of a role of Di-ras2 in the regulation of the caudal serotonergic system. The latter is intriguing, as serotonin has been found to be critically involved in brain development and to impact on ADHD-related traits such as aggression and impulsivity (Azmitia, 2001; Halperin et al, 1994; Lucki, 1998).

We also strived to study a potential functional role of the DIRAS2 rs1412005 risk variant on the systems level, ie, to test for an association with functional deficits known to be frequently present in ADHD representing putative endophenotypes of the disease. Inhibition of inappropriate responses is an often reported deficit associated with ADHD (Willcutt et $a l, 2005$ ) and has the potential to explain a range of overt behavioral symptoms. In the highly structured setting of a laboratory experiment, however, ADHD does not always manifest in behavioral changes in this experiment (ie, increased error rates (Draeger et al, 1986)). Nevertheless, analysis of brain functioning during neuropsychological tasks may reflect subtle deficits underlying response control also in the absence of behavioral consequences (Fallgatter et al, 2005).

The NoGo task requires inhibitory control from the prefrontal cortex and deficits should be reflected in the number of false alarms in the behavioral output (errors of commission). NGA may be an indicator of prefrontal functioning, and in ADHD, a diminished NGA and corresponding impaired inhibitory performance on the Continuous Performance Test has been reported in both children (Fallgatter et al, 2004) and adult samples (Fallgatter et al, 2005).

On the electrophysiological level, DIRAS2 had functional implications in patients afflicted with ADHD. Carrying the risk genotype went along with impaired prefrontal response control, as indicated by the absent anteriorization of the brain's electrical field when a prepared response has to be actively inhibited (NGA). Notably, the DIRAS2 genotype did not affect behavioral outcomes such as errors rates or reaction times in this group. This moderating influence of DIRAS2 is absent in controls, where only the condition (Go vs NoGo), but not genotype (Figure 3) influenced brain activation in accordance with previous publications (Dresler et al, 2010). Response inhibition is closely linked to the right inferior frontal cortex (Aron and Poldrack, 2005), and the origins of the NGA as an electrophysiological correlate of prefrontal function have been traced back to the anterior cingulate cortex (ACC), a brain region that is known for its role in a wide range of cognitive and emotional tasks. Several studies could show structural as well as functional alterations in the ACC of individuals suffering from ADHD (Cubillo et al, 2012). As DIRAS2 is prominently expressed in this brain region (Allen brain atlas, http://www.brain-map.org/) and the promotor polymorphism seems to have an impact on DIRAS2 expression levels, its expressional changes in interaction with structural alterations associated with ADHD ultimately might lead to the development of ADHD.

Taken together, the results from the present study imply that rs1412005 in the DIRAS2 gene is in itself a causal variant, and that it is not only functional on the molecular, but also the inhibition-related brain activation level, the latter specifically in patients suffering from ADHD. This warrants further investigations on the mechanisms mediating between DIRAS2 genotype and ADHD.

\section{FUNDING AND DISCLOSURE}

This study was supported by EU FP7 Aggressotype Consortium (http://www.aggressotype.eu/, grant agreement no. 602805), EU Horizon2020 Marie Sklodowska Curie European Training Program: MiND (http://www.mindproject.eu/, grant agreement no. 643051), EU Horizon2020 consortium CoCA (http://www.coca-project.eu/, grant agreement no. 667302), the German Research Foundation (grant RE1632/5-1), the Interdisciplinary Center for Clinical Research (IZKF), University of Würzburg, grant Z-3/24 and the ECNP Network ADHD across the Lifespan (http:// www.ecnp.eu/projects-initiatives/ECNP-networks.aspx). The authors declare no conflict of interest.

\section{ACKNOWLEDGMENTS}

We thank J. Romanos, A. Boreatti-Hümmer, and M. Heine for their dedicated assistance in patient recruitment. I. Reck and M. Rex-Haffner are credited for excellent technical assistance. Finally, we thank all patients, families, and controls for their participation in this study. We thank the German Academic Exchange Service (DAAD) and the University of Bergen for supporting Dr Landaas' stay in Germany and to thereby enable her to contribute in the DIRAS2 research project.

\section{REFERENCES}

Altmann A, Weber P, Quast C, Rex-Haffner M, Binder EB, Muller-Myhsok B (2011). vipR: variant identification in pooled DNA using R. Bioinformatics 27: i77-i84.

Aron AR, Poldrack RA (2005). The cognitive neuroscience of response inhibition: relevance for genetic research in attentiondeficit/hyperactivity disorder. Biol Psychiatry 57: 1285-1292.

Asherson P, Zhou K, Anney RJ, Franke B, Buitelaar J, Ebstein R et al (2008). A high-density SNP linkage scan with 142 combined subtype ADHD sib pairs identifies linkage regions on chromosomes 9 and 16. Mol Psychiatry 13: 514-521.

Azmitia EC (2001). Modern views on an ancient chemical: serotonin effects on cell proliferation, maturation, and apoptosis. Brain Res Bull 56: 413-424.

Cubillo A, Halari R, Smith A, Taylor E, Rubia K (2012). A review of fronto-striatal and fronto-cortical brain abnormalities in children and adults with attention deficit hyperactivity disorder (ADHD) and new evidence for dysfunction in adults with ADHD during motivation and attention. Cortex 48: 194-215.

David M, Dzamba M, Lister D, Ilie L, Brudno M (2011). SHRiMP2: sensitive yet practical SHort Read Mapping. Bioinformatics 27: 1011-1012.

Dickson SP, Wang K, Krantz I, Hakonarson H, Goldstein DB (2010). Rare variants create synthetic genome-wide associations. PLoS Biol 8: e1000294.

Draeger S, Prior M, Sanson A (1986). Visual and auditory attention performance in hyperactive children: competence or compliance. J Abnorm Child Psychol 14: 411-424.

Dresler T, Ehlis A-C, Heinzel S, Renner TJ, Reif A, Baehne CG et al (2010). Dopamine transporter (SLC6A3) genotype impacts neurophysiological correlates of cognitive response control in 
an adult sample of patients with ADHD. Neuropsychopharmacology 35: 2193-2202.

Fallgatter AJ, Ehlis AC, Rösler M, Strik WK, Blocher D, Herrmann MJ (2005). Diminished prefrontal brain function in adults with psychopathology in childhood related to attention deficit hyperactivity disorder. Psychiatry Res 138: 157-169.

Fallgatter AJ, Ehlis AC, Seifert J, Strik WK, Scheuerpflug P, Zillessen KE et al (2004). Altered response control and anterior cingulate function in attention-deficit/hyperactivity disorder boys. Clin Neurophysiol 115: 973-981.

Faraone SV, Perlis RH, Doyle AE, Smoller JW, Goralnick JJ, Holmgren MA et al (2005). Molecular genetics of attentiondeficit/hyperactivity disorder. Biol Psychiatry 57: 1313-1323.

Franke B, Neale BM, Faraone SV (2009). Genome-wide association studies in ADHD. Hum Genet 126: 13-50.

Gizer IR, Ficks C, Waldman ID (2009). Candidate gene studies of ADHD: a meta-analytic review. Hum Genet 126: 51-90.

Gratton G, Coles MGH (1989). Generalization and evaluation of eye-movement correction procedures. J Psychophysiol 3: 1-50.

Halperin JM, Sharma V, Siever LJ, Schwartz ST, Matier K, Wornell G et al (1994). Serotonergic function in aggressive and nonaggressive boys with attention deficit hyperactivity disorder. Am J Psychiatry 151: 243-248.

Jacob CP, Romanos J, Dempfle A, Heine M, WindemuthKieselbach C, Kruse A et al (2007). Co-morbidity of adult attention-deficit/hyperactivity disorder with focus on personality traits and related disorders in a tertiary referral center. Eur Arch Psychiatry Clin Neurosci 257: 309-317.

Jasper (1958). Report of the committee on methods of clinical examination in electroencephalography: 1957. Electroencephalogr Clin Neurophysiol 10: 370-375.

Kontani K, Tada M, Ogawa T, Okai T, Saito K, Araki Y et al (2002). Di-Ras, a distinct subgroup of ras family GTPases with unique biochemical properties. J Biol Chem 277: 41070-41078.

Lehmann D, Skrandies W (1980). Reference-free identification of components of checkerboard-evoked multichannel potential fields. Electroencephalogr Clin Neurophysiol 48: 609-621.

Lesch KP, Timmesfeld N, Renner TJ, Halperin R, Roser C, Nguyen TT et al (2008). Molecular genetics of adult ADHD: converging evidence from genome-wide association and extended pedigree linkage studies. J Neural Transm 115: 1573-1585.
Li H, Durbin R (2009). Fast and accurate short read alignment with Burrows-Wheeler transform. Bioinformatics 25: 1754-1760.

Lucki I (1998). The spectrum of behaviors influenced by serotonin. Biol Psychiatry 44: 151-162.

Meldrum C, Doyle MA, Tothill RW (2011). Next-generation sequencing for cancer diagnostics: a practical perspective. Clin Biochem Rev 32: 177-195.

Polanczyk G, de Lima MS, Horta BL, Biederman J, Rohde LA (2007). The worldwide prevalence of ADHD: a systematic review and metaregression analysis. Am J Psychiatry 164: 942-948.

Reif A, Nguyen TT, Weissflog L, Jacob CP, Romanos M, Renner TJ et al (2011). DIRAS2 is associated with adult ADHD, related traits, and co-morbid disorders. Neuropsychopharmacology 36: 2318-2327.

Romanos M, Freitag C, Jacob C, Craig DW, Dempfle A, Nguyen TT et al (2008). Genome-wide linkage analysis of ADHD using high-density SNP arrays: novel loci at 5q13.1 and $14 \mathrm{q} 12$. Mol Psychiatry 13: 522-530.

Rosvold HE, Mirsky AF, Sarason I, Bransome ED Jr, Beck LH (1956). A continuous performance test of brain damage. J Consult Psychol 20: 343-350.

Seshadri S, DeStefano AL, Au R, Massaro JM, Beiser AS, KellyHayes $M$ et al (2007). Genetic correlates of brain aging on MRI and cognitive test measures: a genome-wide association and linkage analysis in the Framingham Study. BMC Med Genet 8(Suppl 1): S15.

Wang K, Li M, Hakonarson H (2010). ANNOVAR: functional annotation of genetic variants from high-throughput sequencing data. Nucleic Acids Res 38: e164.

Wennerberg K, Rossman KL, Der CJ (2005). The Ras superfamily at a glance. J Cell Sci 118(Part 5): 843-846.

Willcutt EG, Doyle AE, Nigg JT, Faraone SV, Pennington BF (2005). Validity of the executive function theory of attention-deficit/ hyperactivity disorder: a meta-analytic review. Biol Psychiatry 57: $1336-1346$.

Wray NR, Purcell SM, Visscher PM (2011). Synthetic associations created by rare variants do not explain most GWAS results. PLoS Biol 9: e1000579.

Wylie CJ, Hendricks TJ, Zhang B, Wang L, Lu P, Leahy P et al (2010). Distinct transcriptomes define rostral and caudal serotonin neurons. J Neurosci 30: 670-684.

Supplementary Information accompanies the paper on the Neuropsychopharmacology website (http://www.nature.com/npp) 